\title{
Low Temperature Smoothness of the Pressure for Antiferromagnets and other Models
}

\author{
David Klein \\ Department of Mathematics \\ California State University \\ Northridge, California 91330
}

\author{
Wei-Shih Yang \\ Department of Mathematics \\ Temple University \\ Philadelphia, Pennsylvania 19122
}

\begin{abstract}
We prove under certain conditions on a grandcanonical Hamiltonian that at low temperature the pressure is infinitely differentiable with respect to the inverse temperature and other parameters in the Hamiltonian, when the parameters are chosen so that the number of Gibbs states is at least equal to the number of ground states. Applications are made to Antiferromagnets and hard-core gases.
\end{abstract}

Key Words. Pressure, Differentiability, Gibbs states, Pirogov-Sinai Theory, Cluster Expansion PACS number: 05.20.-y

\section{Introduction}

An important task for classical equilibrium statistical mechanics is to find general mathematical criteria for phase transitions and phase coexistence. One approach to this problem is the general theory of infinite volume Gibbs states, probability measures determined by the DLR equations first given in Refs. 1 and 2. The problem in this context is to describe all limit Gibbs measures for a given Hamiltonian. Results of this type include proofs of the uniqueness or nonuniqueness of Gibbs states for various values of the thermodynamic parameters associated with the system of particles under consideration.

Another approach to the study of phase transitions is to try to locate all singularities of an appropriate thermodynamic function, e.g., the pressure. Phase transitions are then associated with these singularities. In the low temperature region, investigations along these lines have established smoothness or analyticity for Ferromagnetic-type systems, as in Basuev $^{3}$ and the references contained in Slawny ${ }^{4}$. The related question of the smoothness of the low temperature phase diagram has been analyzed by Zahradnik ${ }^{5}$.

In this paper, we establish some connections between these two criteria for phase transitions in the low temperature region. For a fairly general class of Hamiltonians, we give a simple proof that when parameters in the Hamiltonian like external field strengths are chosen so that the number of Gibbs states at low temperature is at least than the number of 
ground states, then the pressure is infinitely differentiable with respect to inverse temperature and these parameters. Our requirement on the number of Gibbs states is not as restrictive as it may appear. It is frequently possible to fix certain parameters while letting the others vary in such a way that smoothness of the pressure is established in the (low temperature) regions of the phase diagram where the number of phases is constant. This is carried out for the antiferromagnet and hard-core gas.

Although Pirogov-Sinai Theory plays a major role here, the parameters we consider are not necessarily associated with fields which split the degeneracy of our Hamiltonians. Examples are given in Section 4. In Section 3 we state and prove our main result, Theorem 3.1. Section 2 summarizes the parts of Pirogov-Sinai Theory which are needed in the proof of Theorem 3.1. We assume that the reader has some familiarity with the method of cluster expansions (c.f. Ref. 6) which is also used in the proof.

\section{Summary of Pirogov-Sinai Theory}

In this section we summarize Pirogov-Sinai Theory $7,4,8$ in a form which will be useful in Sect. 3.

We consider Hamiltonians formally given by

$$
\mathrm{H}(\mathrm{x})=\beta \Sigma_{\Lambda} \phi\left(\mathrm{x}_{\Lambda}\right)
$$

where $\phi$ is a finite range periodic interaction potential, $x_{\Lambda}$ is the restriction of the configuration $\mathrm{x}$ to the subset $\Lambda$ of $Z^{\mathrm{d}}$, and the sum is over finite subsets of $Z^{\mathrm{d}}$. The parameter $\beta$ represents the inverse temperature. For $\mathrm{i} \in \mathrm{Z}^{\mathrm{d}}$ let $\mathrm{x}_{\mathrm{i}}$, the restriction of $\mathrm{x}$ to $\mathrm{i}$, take values in some finite set $\mathrm{X}$. The reference measure on $\mathrm{X}$ is the counting measure. The energy of a finite configuration (with empty boundary conditions) is then given by

$$
\mathrm{H}\left(\mathrm{x}_{\Lambda}\right)=\beta \sum_{\mathrm{A} \subset \Lambda} \phi\left(\mathrm{x}_{\mathrm{A}}\right)
$$

With only minor modifications in what follows, we may assume that $\mathrm{H}$ has a hardcore restriction. In that case, configurations $\mathrm{x}$ and $\mathrm{y}$ below are assumed to be "allowable" in that they satisfy the hard-core restriction. 
It is convenient to use the notion of relative Hamiltonian. Let two configurations $\mathrm{x}$ and $\mathrm{y}$ be equal at all but a finite number of sites in $\mathrm{Z}^{\mathrm{d}}$. In this case we write $\mathrm{x}=\mathrm{y}$ (a.s.).

Define

$$
\mathrm{H}(\mathrm{x} \mid \mathrm{y})=\beta \sum_{\Lambda}\left[\phi\left(\mathrm{x}_{\Lambda}\right)-\phi\left(\mathrm{y}_{\Lambda}\right)\right]
$$

Note that the sum in (2.2) is finite. A configuration $x$ is called a ground state if $H(x \mid y) \geq 0$ for each $\mathrm{y}$ such that $\mathrm{y}=\mathrm{x}$ (a.s.). We consider only periodic ground states and we assume that $\mathrm{H}$ has only a finite number of periodic ground states, $\mathrm{x}^{1}, \ldots, \mathrm{x}^{\mathrm{n}}$.

The specific energy $\mathrm{e}_{\mathrm{x}}(\mathrm{H})$ of a periodic ground state $\mathrm{x}$ is given by

$$
\mathrm{e}_{\mathrm{x}}(\mathrm{H})=\lim _{\Lambda \uparrow \mathrm{Z}^{\mathrm{d}}} \frac{1}{|\Lambda|} \sum_{\mathrm{i} \in \Lambda} \mathrm{U}_{\mathrm{i}}(\mathrm{x})
$$

where the limit may be taken over an increasing sequence of hypercubes and where

$$
\mathrm{U}_{\mathrm{i}}(\mathrm{x})=\beta \sum_{\mathrm{A}: \mathrm{i} \in \mathrm{A}} \frac{1}{|\mathrm{~A}|} \phi\left(\mathrm{x}_{\mathrm{A}}\right)
$$

with | | denoting cardinality.

We note that for any two periodic ground states $\mathrm{x}^{1}$ and $\mathrm{x}^{2}$ of $\mathrm{H}$,

$$
\mathrm{e}_{\mathrm{x}^{1}}(\mathrm{H})=\mathrm{e}_{\mathrm{x}^{2}}(\mathrm{H})
$$

This is proved in Lemma 2.1 of Ref.4.

For $i \in Z^{d}$, let

$$
\mathrm{W}(\mathrm{i})=\left\{\mathrm{j} \in \mathrm{Z}^{\mathrm{d}}:\|\mathrm{i}-\mathrm{j}\| \leq \mathrm{r}\right\}
$$

where \|\| denotes the $\mathrm{L}_{\infty}$--norm of $\mathbf{R}^{\mathrm{d}}$ restricted to $\mathrm{Z}^{\mathrm{d}}$ and $\mathrm{r}$ is a fixed number greater than the radius of interaction of $\phi$ and large enough so that all periodic ground states are uniquely determined by their restrictions to $\mathrm{W}(\mathrm{i})$.

A hypercube $\mathrm{W}(\mathrm{i})$ is said to be an irregular cube of the configuration $\mathrm{x}$ if $\mathrm{x}$ restricted to $\mathrm{W}(\mathrm{i})$ is not equal to any ground state restricted to $\mathrm{W}(\mathrm{i})$. The boundary $\mathrm{B}(\mathrm{x})$ of $\mathrm{x}$ is the union of all irregular cubes of $\mathrm{x}$.

A subset $\Lambda$ of $Z^{\mathrm{d}}$ is connected if $\Lambda$ cannot be written as $\Lambda_{1} \cup \Lambda_{2}$ with $\mathrm{d}\left(\Lambda_{1}, \Lambda_{2}\right)>1$. Here the distance is determined by the $\mathrm{L}_{\infty}$ norm. 
Let $\mathrm{y}=\mathrm{x}^{\mathrm{q}}$ (a.s.) for some $\mathrm{q}=1, \ldots, \mathrm{n}$ and let $\mathrm{M}$ be a connected component of the boundary $\mathrm{B}(\mathrm{y})$. The pair $\left(\mathrm{M}, \mathrm{y}_{\mathrm{M}}\right)$ is a contour of $\mathrm{y}$. A pair $\left(\mathrm{M}, \mathrm{x}_{\mathrm{M}}\right)$ is called a contour if it is the contour of some configuration. $\mathrm{M}$ is called the support of $\Gamma$ and we write $|\Gamma|=|\mathrm{M}|$.

Let $\Gamma=\left(\mathrm{M}, \mathrm{x}_{\mathrm{M}}\right)$ be a contour. Then there exists a unique configuration $\mathrm{x}_{\Gamma}$ such that $\mathrm{x}_{\Gamma}=\mathrm{x}_{\mathrm{M}}$ on $\mathrm{M}$ and $\mathrm{M}=\mathrm{B}(\mathrm{x})$. Also since $|\mathrm{M}|<\infty$, there exists a unique infinite connected component of $\mathrm{M}^{\mathrm{c}}$ which is called the exterior of $\Gamma$, ext $\Gamma$, and the rest of $\mathrm{M}^{\mathrm{c}}$ is called the interior of $\Gamma$, Int $\Gamma$. On each connected component of $\mathrm{M}^{\mathrm{c}}, \mathrm{x}_{\Gamma}$ is equal to one of the ground states. We will write $\Gamma=\Gamma \mathrm{q}$ if $\mathrm{x}_{\Gamma}=\mathrm{x}^{\mathrm{q}}$ on ext $(\Gamma)$. The m-interior, $\operatorname{Int}_{\mathrm{m}} \Gamma$, of $\Gamma$ is the union of components of the interior of $\Gamma$ on which $\mathrm{x}_{\Gamma}=\mathrm{x}^{\mathrm{m}}$. The contour $\Gamma$ is called an exterior contour if it is not contained in the interior of any other contour.

Let $\Lambda$ be a finite subset of $Z^{d}$. Let $R_{q}(\Lambda)$ be the set of all configurations $x$ such that $\mathrm{x}_{\mathrm{i}}=\mathrm{x}_{\mathrm{i}}^{\mathrm{q}}$ for all $\mathrm{i} \in \Lambda^{\mathrm{c}}, \mathrm{d}\left(\mathrm{B}(\mathrm{x}), \Lambda^{\mathrm{c}}\right)>1$, and for any contour $\Gamma$ of $\mathrm{x}$, Int $(\Gamma) \subset \Lambda$. The rarified partition function in volume $\Lambda$ with boundary conditions $\mathrm{x}^{\mathrm{q}}$ for the Hamiltionian $\mathrm{H}$ is defined by

$$
\mathrm{Z}^{\mathrm{q}}(\Lambda \mid \mathrm{H})=\sum_{\mathrm{x} \in \mathrm{R}_{\mathrm{q}}(\Lambda)} \exp \left[-\mathrm{H}\left(\mathrm{x} \mid \mathrm{x}^{\mathrm{q}}\right)\right]
$$

Let $Z_{\Lambda}^{\mathrm{q}}$ denote the usual partition function, i.e.,

$$
\mathrm{Z}_{\Lambda}^{\mathrm{q}}=\sum_{\mathrm{x} \in \Lambda} \exp \left[-\mathrm{H}^{\mathrm{q}}(\mathrm{x})\right]
$$

where the sum is over (allowable) configurations in $\Lambda$ and

$$
\mathrm{H}^{\mathrm{q}}(\mathrm{x})=\sum_{\mathrm{A} \cap \Lambda \neq \varnothing} \phi\left(\mathrm{y}_{\mathrm{A}}\right)
$$

and $\mathrm{y}=\mathrm{x}_{\Lambda}$ on $\Lambda$ and $\mathrm{y}=\mathrm{x}^{\mathrm{q}}$ on $\Lambda^{\mathrm{c}}$. Observe that given a finite set $\Lambda \subset \mathrm{Z}^{\mathrm{d}}$, there is a unique subset $\Lambda^{\prime} \subset \Lambda$ such that

$$
\mathrm{Z}^{\mathrm{q}}(\Lambda \mid \mathrm{H})=\mathrm{Z}_{\Lambda^{\prime}}^{\mathrm{q}} \exp \left[-\mathrm{H}\left(\mathrm{x}_{\Lambda^{\prime}}^{\mathrm{q}}\right)\right]
$$

As in Ref. 8, we write $\Gamma \mathrm{q} \subset \Lambda$ if supp $\Gamma \mathrm{q} \subset \Lambda$, dist $\left(\operatorname{supp} \Gamma \mathrm{q}, \Lambda^{\mathrm{c}}\right)>1$, and $\operatorname{Int} \Gamma \mathrm{q} \subset \Lambda$. Two subsets of $Z^{d}$ are said to be far apart if the $\mathrm{L}_{\infty}$ distance between them is greater than one. Let $\mathrm{C}_{\mathrm{q}}(\Lambda)$ be the ensemble whose elements are finite sets of contours $\left\{\Gamma_{1}^{\mathrm{q}}, \ldots, \Gamma_{\mathrm{m}}^{\mathrm{q}}\right\}, \Gamma_{\mathrm{i}}^{\mathrm{q}} \subset$ $\Lambda$ with supports pairwise far apart. These contours $\left\{\Gamma_{1}^{q}, \ldots, \Gamma_{\mathrm{m}}^{\mathrm{q}}\right\}$ in $\mathrm{C}_{\mathrm{q}}(\Lambda)$ are in general not contours of a configuration $\mathrm{x}$. Each contour has boundary condition $\mathrm{x}$. 
Let $\mathrm{F}_{\mathrm{q}}$ be a nonnegative functional defined on $\mathrm{C}_{\mathrm{q}}(\Lambda)$. The contour rarified partition function in volume $\Lambda$ is defined by

$$
\mathrm{Z}\left(\Lambda \mid \mathrm{F}_{\mathrm{q}}\right)=\sum \exp \left[-\sum_{\mathrm{i}} \mathrm{F}_{\mathrm{q}}\left(\Gamma_{\mathrm{i}}^{\mathrm{q}}\right)\right]
$$

where the summation is over all possible $\left\{\Gamma_{1}^{q}, \ldots, \Gamma_{\mathrm{m}}^{\mathrm{q}}\right\}$ in $\mathrm{C}_{\mathrm{q}}(\Lambda)$.

A functional $\mathrm{F}_{\mathrm{q}}$ is called a $\tau$-funtional if

$$
\mathrm{F}_{\mathrm{q}}(\Gamma \mathrm{q}) \geq \tau|\Gamma \mathrm{q}| .
$$

In order to apply Pirogov-Sinai theory, we need to check Peierls' condition for $\mathrm{H}$, namely, there exists a positive constant $\rho$ such that for any periodic ground state $\mathrm{x} q$, and any $\mathrm{x}$ such that $\mathrm{x}=\mathrm{x}^{\mathrm{q}}($ a.s. $)$

$$
H(x \mid x q) \geq \rho|B(x)|
$$

The following Lemma is a consequence of Pirogov-Sinai Theory.

Lemma 2.1 Let $H$ have $n$ distinct periodic ground states. Assume that for all $\beta$ sufficiently large, $H$ has $n$ distinct Gibbs states which are limits of finite volume Gibbs measures with boundary conditions equal respectively to the periodic ground states. Each ground state is then associated with one Gibbs state. Assume that there are external fields $H_{1}, \ldots, H_{n-1}$ which completely split the $n$-fold degeneracy of $H$ in the sense of Pirogov-Sinai. Then for $\beta$ sufficiently large there exists a number $\tau$ and $\tau$-functionals $F_{q}, q=1, \ldots, n$ such that

$$
\mathrm{Zq}(\Lambda \mid \mathrm{H})=\mathrm{Z}\left(\Lambda \mid \mathrm{F}_{\mathrm{q}}\right)
$$

for all $q$ and $\Lambda$. Moreover each $F_{q}$ satisfies

$$
\mathrm{F}_{\mathrm{q}}(\Gamma \mathrm{q})=\mathrm{H}(\Gamma \mathrm{q})+\sum_{\mathrm{k}=1}^{\mathrm{n}} \log \mathrm{Z}\left(\operatorname{Int}_{\mathrm{k}} \Gamma \mathrm{q} \mid \mathrm{F}_{\mathrm{q}}\right)-\log \mathrm{Z}\left(\operatorname{Int}_{\mathrm{k}} \Gamma \mathrm{q} \mid \mathrm{F}_{\mathrm{k}}\right)
$$

where $\mathrm{H}(\Gamma \mathrm{q})=\mathrm{H}\left(\mathrm{x}_{\Gamma^{q}} \mid \mathrm{x}^{\mathrm{q}}\right)$.

The proof of Lemma 2.1 follows easily from the Main Theorem B and the proof of Proposition 2.6 in Ref. 8 . We note that $\tau$ may be chosen arbitrarily large by choosing $\beta$ sufficiently large. 
Remark 2.1 The existence of the external fields $\mathrm{H}_{1}, \ldots, \mathrm{H}_{\mathrm{n}-1}$ is a technical requirement which allows us to apply Pirogov-Sinai Theory. We are only interested in the case $\mu_{i} \equiv 0$ for the Hamiltonian $\mathrm{H}^{\prime}=\mathrm{H}+\mu_{1} \mathrm{H}_{1}+\cdots+\mu_{\mathrm{n}-1} \mathrm{H}_{\mathrm{n}-1}$. With the hypotheses of Lemma 2.1, $\mu_{\mathrm{i}} \equiv 0$ corresponds to the case of $\mathrm{n}$ (the maximal number) of coexisting phases for $\mathrm{H}^{\prime}$ at low temperature and small values of $\left\{\mu_{i}\right\}$.

\section{Differentiability of the Pressure}

Assume that $\mathrm{H}$ depends on parameters $\lambda_{1}, \ldots, \lambda_{\mathrm{m}}$, where $\lambda_{1}=\beta$, the inverse temperature. The remaining parameters might correspond to external field strengths or to variables which parameterize a hypersurface in the phase diagram (c.f. Sect. 4). In this section we use Lemma 2.1 to prove with certain hypotheses that the pressure corresponding to $\mathrm{H}$ is a $\mathrm{C}^{\infty}$ function of these parameters.

With the notation of Sect. 2, the pressure $\mathrm{P}$ for $\mathrm{H}$ is defined by

$$
\mathrm{P}\left(\lambda_{1}, \ldots, \lambda_{\mathrm{m}}\right)=\lim _{\Lambda \uparrow Z^{\mathrm{d}}} \frac{1}{|\Lambda|} \mathrm{Z}_{\Lambda}^{\varnothing}
$$

where $\varnothing$ indicates empty boundary conditions. Under general conditions (see, for example, Preston $^{8}$ ) which are satisfied here, $\varnothing$ may be replaced by an arbitrary boundary condition in (3.1). We exploit this fact in the proof of Theorem 3.1 below.

In what follows we use the notation $\{\cdot\rangle_{\Lambda}^{q}$ for expectation with respect to the finite volume Gibbs state for $\mathrm{H}$ in volume $\Lambda$ with boundary configuration $\mathrm{x}$.

Theorem 3.1 Let $H$ be a finite range periodic Hamiltonian depending on parameters $\lambda_{1}, \ldots, \lambda_{m}$ as described above. Let intervals $I_{1}, \ldots, I_{m}$ be given where $I_{1}=\left(\beta_{1}, \infty\right)$. For $\lambda_{k}, k=1, \ldots, m$, in any finite open subinterval of $I_{k}$ assume that $H$ satisfies the following conditions:

a) H satisfies Peierls' condition

b) H has exactly $n$ periodic ground states $x^{1}, \ldots, x^{n}$ 
c) H has n extremal Gibbs states which are limits of finite volume Gibbs measures with boundary configurations given respectively by $x^{l}, \ldots, x^{n}$

d) $\max _{\mathrm{x} \subset \Lambda}\left|\frac{\partial}{\partial \lambda_{\mathrm{k}}} \mathrm{H}\left(\mathrm{x}_{\Lambda}\right)\right| \leq \mathrm{c}_{1}|\Lambda|$ for some constant $c_{l}$ and all finite $\Lambda \subset Z^{d}$.

e) For any ground state configuration $x q$ and any $N$, there exists a constant $c(N)$ such that $\left|\frac{\partial^{N}}{\partial \lambda_{\mathrm{k}}^{N}} \mathrm{H}\left(\Gamma^{\mathrm{q}}\right)\right|=\left|\frac{\partial^{\mathrm{N}}}{\partial \lambda_{\mathrm{k}}^{\mathrm{N}}} \mathrm{H}\left(\mathrm{x}_{\Gamma^{q}} \mid \mathrm{x}^{\mathrm{q}}\right)\right| \leq \mathrm{c}(\mathrm{N})\left|\Gamma^{\mathrm{q}}\right|^{\mathrm{N}}$ and $\left|\frac{\partial^{\mathrm{N}}}{\partial \lambda_{\mathrm{k}}^{\mathrm{N}}} \mathrm{H}\left(\mathrm{x}_{\Lambda}^{\mathrm{q}}\right)\right| \leq \mathrm{c}(\mathrm{N})|\Lambda|$ for any finite $\Lambda$. Then for any $k=1, \ldots$, , $m$ there exists $\beta_{0}$ such that $\frac{\partial^{\mathrm{N}} \mathrm{P}}{\partial \lambda_{\mathrm{k}}^{\mathrm{N}}}$ exists for all $N=1,2, \ldots$, when $\lambda_{k} \in I_{k}$ provided $\beta \equiv \lambda_{1}>\beta_{0}$.

Remark 3.1 We note that condition c) of Theorem 3.1 does not require the number of Gibbs states to equal the number of ground states; the number of Gibbs states may exceed the number of ground states. Thus, for example, the theorem applies to Hamiltonians with the parameters chosen so that there is only one ground state, but possibly more than one Gibbs state. However, examples exist where a) and b) of Theorem 3.1 are satisfied but not c) as shown in the introduction of Ref. 4. In an interesting example in the introduction of Ref. 4, a Hamiltonian and its low temperature phase diagram are shown. There are points on the phase diagram corresponding to i) three ground states and one Gibbs state ii) one ground state and two Gibbs states iii) two ground states and three Gibbs states, etc. Proof. Let $\left\{\Lambda_{\mathrm{n}}\right\}$ be a sequence of volumes converging to $\mathrm{Z}^{\mathrm{d}}$ and for a fixed value of $\mathrm{q}$ let $\mathrm{f}_{\mathrm{n}}=\frac{1}{|\Lambda|} \log \mathrm{Z}_{\Lambda_{\mathrm{n}}}^{\mathrm{q}}$. If $\left\{\frac{\partial \mathrm{f}_{\mathrm{n}}}{\partial \lambda_{\mathrm{k}}}\right\}$ is a family of equicontinuous functions in $\lambda_{\mathrm{k}}$ which are uniformly bounded for all $\lambda_{\mathrm{k}}$ in any open subinterval of $\mathrm{I}_{\mathrm{k}}$ then by the Arzela-Ascolli Theorem, there exists a subsequence $\left\{\frac{\partial f_{n_{m}}}{\partial \lambda_{k}}\right\}$ uniformly convergent in any open subinterval of $\mathrm{I}_{\mathrm{k}}$. This implies that $\mathrm{P}$ is differentiable with respect to $\lambda_{\mathrm{k}}$ on $\mathrm{I}_{\mathrm{k}}$ and

$$
\frac{\partial P}{\partial \lambda_{k}}=\lim _{m \rightarrow \infty} \frac{\partial f_{n_{m}}}{\partial \lambda_{k}}
$$


Similarly, by induction, if $\left\{\frac{\partial^{N} f_{n}}{\partial \lambda_{k}^{N}}\right\}$ is uniformly bounded in any open subinterval of $I_{k}$ for all $\mathrm{N}=1,2, \ldots$, then it follows that $\mathrm{P}$ is infinitely differentiable in $\lambda_{\mathrm{k}}$.

Thus it suffices to show that for $\beta$ in a finite open interval with $\beta>\beta_{0}$ (for some $\beta_{0}$ ) and $\lambda_{\mathrm{k}}$ in an open subinterval of $\mathrm{I}_{\mathrm{k}}$

$$
\frac{1}{|\Lambda|}\left|\frac{\partial^{\mathrm{N}} \log Z_{\Lambda^{\prime}}^{\mathrm{q}}}{\partial \lambda_{\mathrm{k}}^{\mathrm{N}}}\right| \leq \mathrm{M}_{\mathrm{N}}^{\prime}
$$

for all hypercubes $\Lambda^{\prime}$ with side length sufficiently large and some constants $\mathrm{M}_{\mathrm{N}}{ }_{\mathrm{N}}$ which may depend on the subintervals of $I_{1}$ and $I_{k}$.

We next modify (3.2) by using equation (2.4). By condition e) of the Theorem,

$$
\frac{1}{|\Lambda|} \frac{\partial^{\mathrm{N}}}{\partial \lambda_{\mathrm{k}}^{\mathrm{N}}} \log \exp \left\{\mathrm{H}\left(\mathrm{x}_{\Lambda^{\mathrm{q}}}^{\mathrm{q}}\right)\right\}=\frac{1}{|\Lambda|} \frac{\partial^{\mathrm{N}}}{\partial \lambda_{\mathrm{k}}^{\mathrm{N}}} \mathrm{H}\left(\mathrm{x}_{\Lambda^{\mathrm{q}}}^{\mathrm{q}}\right)
$$

is bounded for each $N$ and all $\Lambda^{\prime}$. Also $\frac{\left|\Lambda_{\mathrm{i}}^{\prime}\right|}{\left|\Lambda_{\mathrm{i}}\right|} \rightarrow 1$ as $\mathrm{i} \rightarrow \infty$ for any sequence $\Lambda_{\mathrm{i}} \uparrow \mathrm{Z}^{\mathrm{d}}$. To prove the Theorem it therefore suffices to prove that there exists a $\beta_{0}$ such that for $\beta$ in any open subinterval of $\left(\beta_{0}, \infty\right)$,

$$
\frac{1}{|\Lambda|}\left|\frac{\partial^{\mathrm{N}} \log Z^{\mathrm{q}}(\Lambda \mid \mathrm{H})}{\partial \lambda_{\mathrm{k}}^{\mathrm{N}}}\right| \leq \mathrm{M}_{\mathrm{N}}
$$

for all $\lambda_{k}$ in a finite open subinterval of $I_{k}$, all $\Lambda$, and some constants $M_{N}$ which may depend on the subintervals, and all $\mathrm{N}=1,2, \ldots$.

By (2.6) there exists $\beta_{0}$ such that if $\beta>\beta_{0}$ then

$$
\begin{aligned}
\mathrm{Zq}(\Lambda \mid \mathrm{H}) & =\mathrm{Z}\left(\Lambda \mid \mathrm{F}_{\mathrm{q}}\right) \\
& =\sum_{\substack{\left\{\Gamma_{1}^{\mathrm{q}}, \ldots, \Gamma_{\mathrm{m}}^{\mathrm{q}}\right\} \in \mathrm{C}(\Lambda)\\
}} \exp \left[-\sum_{\mathrm{i}=1}^{\mathrm{m}} \mathrm{F}_{\mathrm{q}}\left(\Gamma_{\mathrm{i}}^{\mathrm{q}}\right)\right] \\
& =\sum_{\mathrm{m}=0}^{\infty} \frac{1}{\mathrm{~m} !} \sum_{\Gamma_{1}^{\mathrm{q}}, \ldots, \Gamma_{\mathrm{m}}^{\mathrm{q}} \subset \Lambda} \psi(1, \ldots, \mathrm{m}) \exp \left[-\sum_{\mathrm{i}=1}^{\mathrm{m}} \mathrm{F}_{\mathrm{q}}\left(\Gamma_{\mathrm{i}}^{\mathrm{q}}\right)\right]
\end{aligned}
$$

where

$$
\psi(1, \ldots, \mathrm{m})=\exp \left[-\sum_{1 \leq \mathrm{i}<\mathrm{j} \leq \mathrm{m}} \mathrm{V}(\mathrm{i}, \mathrm{j})\right]
$$


and $\mathrm{V}(\mathrm{i}, \mathrm{j})=\infty$ if $\Gamma_{\mathrm{i}}^{\mathrm{q}}$ and $\Gamma_{\mathrm{j}}^{\mathrm{q}}$ are not far apart and $\mathrm{V}(\mathrm{i}, \mathrm{j})=0$ if $\Gamma_{\mathrm{i}}^{\mathrm{q}}$ and $\Gamma_{\mathrm{j}}^{\mathrm{q}}$ are far apart. As in Mayer's expansion 6 , let

$$
\psi_{\mathrm{c}}(1, \ldots, \mathrm{m})=\sum_{\gamma} \prod_{(\mathrm{i}, \mathrm{j}) \in \gamma}\{\exp [-\mathrm{V}(\mathrm{i}, \mathrm{j})]-1\}
$$

where $\gamma$ is a connected graph ${ }^{6}$ on $\{1, \ldots, \mathrm{m}\}$.

Then

$$
\log \mathrm{Z}\left(\Lambda \mid \mathrm{F}_{\mathrm{q}}\right)=\sum_{\mathrm{m}=1}^{\infty} \frac{1}{\mathrm{~m} !} \sum_{\Gamma_{1}^{q}, \ldots, \Gamma_{\mathrm{m}}^{q} \subset \Lambda} \psi_{\mathrm{c}}(1, \ldots, \mathrm{m}) \exp \left[-\sum_{\mathrm{i}=1}^{\mathrm{m}} \mathrm{F}_{\mathrm{q}}\left(\Gamma_{\mathrm{i}}^{\mathrm{q}}\right)\right]
$$

and

$$
\frac{1}{|\Lambda|} \frac{\partial^{\mathrm{N}}}{\partial \lambda_{\mathrm{k}}^{\mathrm{N}}} \log \mathrm{Z}\left(\Lambda \mid \mathrm{F}_{\mathrm{q}}\right)=\frac{1}{|\Lambda|} \sum_{\mathrm{m}=1}^{\infty} \frac{1}{\mathrm{~m} !} \sum_{\Gamma_{1}^{\mathrm{q}}, \ldots, \Gamma_{\mathrm{m}}^{\mathrm{q}} \subset \Lambda} \psi_{\mathrm{c}}(1, \ldots, \mathrm{m}) \frac{\partial^{\mathrm{N}}}{\partial \lambda_{\mathrm{k}}^{\mathrm{N}}} \exp \left[-\sum_{\mathrm{i}=1}^{\mathrm{m}} \mathrm{F}_{\mathrm{q}}\left(\Gamma_{\mathrm{i}}^{\mathrm{q}}\right)\right]
$$

Let $\mathrm{f}=-\sum_{\mathrm{i}=1}^{\mathrm{m}} \mathrm{F}_{\mathrm{q}}\left(\Gamma_{\mathrm{i}}^{\mathrm{q}}\right)$. Then $\frac{\partial^{\mathrm{N}}}{\partial \lambda_{\mathrm{k}}^{\mathrm{N}}} \operatorname{expf}$ is a sum of at most $\mathrm{N}$ ! terms and each term is of the form $e^{f} \frac{\partial^{i_{1}} f}{\partial \lambda_{k}^{i_{L}}} L \frac{\partial^{i_{L}} f}{\partial \lambda_{k}^{i_{L}}}$ with $i_{1}+\cdots+i_{L}=N, i_{j} \geq 1$ for all $j=1, \ldots, L$. We use formula (2.7) to compute $\frac{\partial^{\prime}}{\partial \lambda_{\mathrm{k}}^{\prime}} \mathrm{F}_{\mathrm{q}}\left(\Gamma^{\mathrm{q}}\right)$. It is necessary to first estimate

$$
\frac{\partial^{\prime}}{\partial \lambda_{\mathrm{k}}^{\prime}} \log \mathrm{Z}\left(\Lambda \mid \mathrm{F}_{\mathrm{q}}\right)=\frac{\partial^{\prime}}{\partial \lambda_{\mathrm{k}}^{\prime}} \log \left[\mathrm{Z}_{\Lambda^{\mathrm{q}}}^{\mathrm{q}} \cdot \exp \left\{-\mathrm{H}\left(\mathrm{x}_{\Lambda^{\mathrm{q}}}^{\mathrm{q}}\right)\right]\right.
$$

For $I \geq 1$ the right side of (3.7) is a sum of at most $2 \mid \mathrm{I}$ ! terms of the form

$$
\left.\left.\pm\left\langle\left(\frac{\partial}{\partial \lambda_{k}} H\right)^{k_{1}}\right\rangle_{\Lambda^{\prime}}^{q} /\left(\frac{\partial}{\partial \lambda_{k}} H\right)^{k_{2}}\right\rangle_{\Lambda^{\prime}}^{q} L /\left(\frac{\partial}{\partial \lambda_{k}} H\right)^{k_{1}}\right\rangle_{\Lambda^{\prime}}^{q}
$$

where $\mathrm{k}_{1}+\cdots+\mathrm{k}_{\mathrm{t}}=\mathrm{l}, \mathrm{i}_{\mathrm{j}} \geq 1$ for $\mathrm{j}=1, \ldots, \mathrm{t}$.

By condition d),

$$
\left|\frac{\partial^{\prime}}{\partial \lambda_{\mathrm{k}}^{\prime}} \log \mathrm{Z}\left(\Lambda \mid \mathrm{F}_{\mathrm{q}}\right)\right| \leq\left. 2^{\prime}\left|! \mathrm{c}_{1}^{\prime}\right| \Lambda\right|^{\prime} \equiv \mathrm{c}_{2}|\Lambda|^{\prime}
$$

Combining (3.9) and (2.7) with condition e) gives

$$
\left|\frac{\partial^{\prime}}{\partial \lambda_{\mathrm{k}}^{\prime}} \mathrm{F}_{\mathrm{q}}\left(\Gamma^{\mathrm{q}}\right)\right| \leq \mathrm{c}(\mathrm{I})\left|\Gamma^{\mathrm{q}}\right|^{\prime}+\mathrm{nc}_{2}\left|\operatorname{Int} \Gamma^{\mathrm{q}}\right|^{\prime} \leq \mathrm{c}(\mathrm{I})\left|\Gamma^{\mathrm{q}}\right|^{\mathrm{I}}+\mathrm{c}_{3}\left|\Gamma^{\mathrm{q}}\right|^{\mathrm{dl}} \leq \mathrm{c}_{4}\left|\Gamma^{\mathrm{q}}\right|^{\mathrm{dl}}
$$

for some geometric constants $\mathrm{c}_{3}$ and $\mathrm{c}_{4}$. Hence

$$
\left|\frac{\partial^{\prime} \mathrm{f}}{\partial \lambda_{\mathrm{k}}^{\prime}}\right| \leq \mathrm{c}_{4} \sum_{\mathrm{i}=1}^{\mathrm{m}}\left|\Gamma_{\mathrm{i}}^{\mathrm{q}}\right|^{\mathrm{dl}} \leq \mathrm{c}_{4}\left(\sum_{\mathrm{i}=1}^{\mathrm{m}}\left|\Gamma_{\mathrm{i}}^{\mathrm{q}}\right|\right)^{\mathrm{d} /}
$$

and 


$$
\begin{aligned}
&\left|\frac{\partial^{\mathrm{N}} \mathrm{e}^{\mathrm{f}}}{\partial \lambda_{\mathrm{k}}^{\mathrm{N}}}\right| \leq \mathrm{e}^{\mathrm{f}} \mathrm{N} ! \mathrm{c}_{4}\left(\sum_{\mathrm{i}=1}^{\mathrm{m}}\left|\Gamma_{\mathrm{i}}^{\mathrm{q}}\right|\right)^{\mathrm{dN}} \\
& \quad \leq \mathrm{c}_{4} \mathrm{e}^{\mathrm{f}} \mathrm{d} !(\mathrm{N} !)^{2} \exp \left(\sum_{\mathrm{i}=1}^{\mathrm{m}}\left|\Gamma_{\mathrm{i}}^{\mathrm{q}}\right|\right) \equiv \mathrm{c}_{5} \mathrm{e}^{\mathrm{f}} \exp \left(\sum_{\mathrm{i}=1}^{\mathrm{m}}\left|\Gamma_{\mathrm{i}}^{\mathrm{q}}\right|\right)
\end{aligned}
$$

Combining (3.6), (3.12), and using the fact that $\mathrm{F}_{\mathrm{q}}$ is a $\tau$-functional gives

$$
\frac{1}{|\Lambda|}\left|\frac{\partial^{N}}{\partial \lambda_{\mathrm{k}}^{\mathrm{N}}} \log \mathrm{Z}\left(\Lambda \mid \mathrm{F}_{\mathrm{q}}\right)\right| \leq \frac{\mathrm{c}_{5}}{|\Lambda|} \sum_{\mathrm{m}=1}^{\infty} \frac{1}{\mathrm{~m} !} \sum_{\Gamma_{1}^{q}, \ldots, \Gamma_{\mathrm{m}}^{\mathrm{q}} \subset \Lambda}\left|\psi_{\mathrm{c}}(1, \ldots, \mathrm{m})\right| \exp \left[(1-\tau) \sum_{\mathrm{i}=1}^{\mathrm{m}}\left|\Gamma_{\mathrm{i}}^{\mathrm{q}}\right|\right]
$$

Now using Penrose tree graph bounds and standard arguments 6 , it follows that (3.13) is bounded by a constant $\mathrm{M}_{\mathrm{N}}$ if

$$
\sum_{\Gamma^{q}: 0 \in \Gamma^{q}} \mathrm{e}^{-(\tau-2)\left|\Gamma^{q}\right|}<1
$$

Since $\tau$ may be chosen arbitrarily large by choosing $\beta$ sufficiently large, (3.14) holds if $\beta \geq \beta_{0}$ for some $\beta_{0}>0$. The proof is completed by combining (3.13) with (3.3).

The following is a corollary to the proof of Theorem 3.1.

Corollary 3.1 Assume all of the hypotheses of Theorem 3.1 except that condition e) holds only for $N=1,2, \ldots, M+1$. Then the pressure is $M$ times differentiable with respect to $\lambda_{k}$ for $\beta>\beta_{0}$.

\section{Examples}

In this section we give some examples and applications of Theorem 3.1.

A) Ferromagnetic Ising Model with $\mathrm{d} \geq 2$

The purpose of this example is just to illustrate Theorem 3.1. We note that low temperature analyticity in $\beta$ is well-known; references are given in Slawny ${ }^{4}$ (see also Ref. 3). Let

$$
H(x)=\beta J \sum_{|i-j| l=1} x_{i} x_{j}-\beta h \sum_{i} x_{i}
$$

with $\mathrm{J}<0$ and $\mathrm{x}_{\mathrm{i}}= \pm 1$ and where $|\cdot|$ denotes the Euclidean norm. Then $\mathrm{H}$ satisfies Peierls' condition for all $\beta=\lambda_{1}$, and $h=\lambda_{2}$. The Hamiltonian $H_{1}=\beta \sum_{i} x_{i}$ completely splits the 
degeneracy of $\mathrm{H}$. If $\mathrm{h}=0, \mathrm{H}$ has two periodic ground states and otherwise one. The remaining hypotheses of Theorem 3.1 are easily checked. The following Corollary holds.

Corollary 4.1 There exists $\beta_{0}(h)$ depending on $h$ such that if $\beta>\beta_{0}(h)$ and

a) $h=0$, then the pressure is infinitely differentiable with respect to $\beta$.

b) $h \neq 0$, then the pressure is infinitely differentiable with respect to $\beta$ and with respect to $h$.

We note that Lebowitz and Martin-Lof ${ }^{10}$ proved that the pressure is not differentiable with respect to $\mathrm{h}$ at $\mathrm{h}=0$ for all values of the temperature below the critical temperature.

B) Antiferromagnet, $d \geq 2$

Let

$$
\mathrm{H}(\mathrm{x})=\beta \sum_{|\mathrm{i}-\mathrm{j}|=1} \mathrm{x}_{\mathrm{i}} \mathrm{x}_{\mathrm{j}}-\beta \mathrm{h} \sum_{\mathrm{i}} \mathrm{x}_{\mathrm{i}}
$$

with $x_{i}= \pm 1$. Note that the term $\beta h \sum_{i} x_{i}$ in (4.2) does not split the degeneracy of the Hamiltonian consisting of the first term alone. $\mathrm{H}(\mathrm{x})$ has two ground states if $|\mathrm{h}|<2 \mathrm{~d}$ and one ground state if $|\mathrm{h}|>2 \mathrm{~d}$. If $|\mathrm{h}|<2 \mathrm{~d}$, a perturbation Hamiltonian and coupling constant $\beta \mu$ which split the degeneracy of (4.2) is $\beta \mu \sum_{i}$ even $x_{i}$, where the sum is over sites in $\mathbf{Z}^{\mathrm{d}}$, the sum of whose components is even. The coefficient $\mu$ is of little physical interest and we consider only the case $\mu=0$. It can be shown that (for $\mu=0) \mathrm{H}(\mathrm{x}$ ) has exactly two ground states and at least two Gibbs states for all $|\mathrm{h}|<2 \mathrm{~d}$ and for $\beta>\beta_{0}(\mathrm{~h})$ where $\beta_{0}(\mathrm{~h})$ may be chosen to depend only on $\min (|\mathrm{h}-2 \mathrm{~d}|,|\mathrm{h}+2 \mathrm{~d}|)$. For a discussion and further references on this we refer the reader to Ref. 11. Analysis of the case $|\mathrm{h}|>2 \mathrm{~d}$ is straightforward. As to the differentiability of the pressure with respect to $\beta$ and $h$ we have the following corollary to Theorem 3.1. 
Corollary 4.2 Let $P$ be the pressure for (4.2). Then if $|h| \neq 2 d$, there exists $\beta_{0}(h)$ which depends only on $\min (|h-2 d|,|h+2 d|)$ such that $P$ is infinitely differentiable with respect to $h$ for $\beta>\beta_{0}(h)$ and infinitely differentiable with respect to $\beta$ for $\beta>\beta_{0}(h)$.

C) Hard-Core Gas, d $\geq 2$

In this example,

$$
\mathrm{H}(\mathrm{x})=\beta \sum_{\mathrm{i}} \mathrm{x}_{\mathrm{i}}
$$

where $\mathrm{x}_{\mathrm{i}}=0$ or 1 and $\mathrm{H}$ is restricted to allowable configurations. A configuration $\mathrm{x}$ is allowable if $x_{i} x_{j}=0$ whenever the Euclidean distance between $i$ and $j$ equals one.

Corollary 4.3 Let $P$ be the pressure for (4.3). Then there exists $\beta_{0}>0$ such if $\beta>\beta_{0}$, then $P$ is infinitely differentiable with respect to $\beta$.

D) Fisher Antiferromagnet, $d=2$

Let

$$
\mathrm{H}(\mathrm{x})=\beta \sum_{(\mathrm{i}, \mathrm{j})} \mathrm{J}_{\mathrm{ij}} \mathrm{x}_{\mathrm{i}} \mathrm{x}_{\mathrm{j}}-\beta \mathrm{h} \sum_{\mathrm{i}} \mathrm{x}_{\mathrm{i}}
$$

where the sum is over pairs $(i, j), x_{i}= \pm 1$, and $J_{i j}$ equals 1 for nearest neighbor pairs $(i, j), J_{i j}$ is a negative constant when $|\mathrm{i}-\mathrm{j}|=\sqrt{ } 2$, and $\mathrm{J}_{\mathrm{ij}}=0$ otherwise. This Hamiltonian was analyzed by Pirogov and Sinai ${ }^{7}$. In order to formulate Corollary 4.4 we repeat some of their analysis. For simplicity, we consider only $h \geq 0$. The case $h \leq 0$ is similar.

Consider the Hamiltonian

$$
\mathrm{H}^{1}(\mathrm{x})=\beta \sum_{(\mathrm{i}, \mathrm{j})} \mathrm{J}_{\mathrm{ij}} \mathrm{x}_{\mathrm{i}} \mathrm{x}_{\mathrm{j}}-4 \beta \sum_{\mathrm{i}} \mathrm{x}_{\mathrm{i}}
$$

$\mathrm{H}^{1}(\mathrm{x})$ satisfies Peierls' condition and has three ground states

$$
\begin{aligned}
& \mathrm{x}^{1}{ }_{\mathrm{i}}=(-1)^{|\mathrm{i}|} \\
& \mathrm{x}^{2}{ }_{\mathrm{i}}=(-1)^{|\mathrm{i}|+1} \\
& \mathrm{x}^{3}{ }_{\mathrm{i}}=1
\end{aligned}
$$

where $|\mathrm{i}|=\left|\left(\mathrm{i}_{1}, \mathrm{i}_{2}\right)\right|=\left|\mathrm{i}_{1}\right|+\left|\mathrm{i}_{2}\right|$. Let $\mathrm{H}_{1}$ and $\mathrm{H}_{2}$ be given by 


$$
\mathrm{H}_{1}=\beta \sum_{\mathrm{i}: \mathrm{i} \mid \mathrm{i} \text { odd }} \mathrm{x}_{\mathrm{i}} \quad \mathrm{H}_{2}=\beta \sum_{\mathrm{i}:|\mathrm{i}| \mathrm{even}} \mathrm{x}_{\mathrm{i}}
$$

Then clearly $\mu_{1} \mathrm{H}_{1}+\mu_{2} \mathrm{H}_{2}$ completely splits the degeneracy of $\mathrm{H}^{1}$.

It follows from Pirogov-Sinai Theory that there exist $\mu_{1}(\beta)$ and $\mu_{2}(\beta)$ such that

$$
H^{1}+\mu_{1}(\beta) H_{1}+\mu_{2}(\beta) H_{2}
$$

has three Gibbs measures, no two of which are a convex combination of the third, when $\beta$ is sufficiently large. Symmetry arguments show that $\mu_{1}(\beta)=\mu_{2}(\beta)$. Referring to the common value of

$\mu_{1}(\beta)-4$ and $\mu_{2}(\beta)-4$ as $h(\beta)$, define

$$
H^{2}(x)=\beta \sum_{(i, j)} J_{i j} x_{i} x_{j}-h(\beta) \sum_{i} x_{i}
$$

The following Corollary follows from Corollary 3.1.

Corollary 4.4 Let $P$ be the pressure for $H^{2}$ given by (4.7). Suppose that $h(\beta)$ is $n$ times continuously differentiable with respect to $\beta$ for all $\beta$ sufficiently large (possibly depending on $n)$. Then there exists a $\beta_{0}$ such that if $\beta>\beta_{0}, P$ is $n$ times differentiable with respect to $\beta$.

Remark 4.1 Applying the results of Zahradnik on the analyticity of the phase diagram ${ }^{5}$, it follows that $h(\beta)$ is an analytic function. Thus the hypothesis that $h(\beta)$ is $n$ times differentiable may be removed in Corollary 4.4 .

Referring back to $\mathrm{H}$ defined by (4.4), it is possible to show that the pressure for $\mathrm{H}$ is infinitely differentiable with respect to $h$ and $\beta$, for large $\beta$, at values of $h$ such that $\mathrm{h}>\max [\mathrm{h}(\beta), 4]$ or $0 \leq \mathrm{h}<\min [\mathrm{h}(\beta), 4]$. This follows from Theorem 3.1 and an analysis similar to the one given above. 


\section{Acknowledgement}

One of us (W.-S. Y.) was partially supported during the course of this research by NSF grant DMS 8902123.

\section{References}

1. Dobrushin, R.L. "The description of a random field by means of conditional probabilities and conditions on its regularity." Theor. Probab. Appl. 13, 197-224 (1968).

2. Lanford, O.E.III, Ruelle, D. "Observables at infinity and states with short range correlations in statistical mechanics." Commun. Math. Phys. 13, 194-215 (1969).

3. Basuev, A. G. "Mayer Expansions for gas of contours at low temperatures and in arbitrary external fields for the multicomponent Ising model." Toer. Mat. Fiz. 58, 261 (1984).

4. Slawny, J. "Low-temperature properties of classical lattice systems: phase transitions and phase diagrams" In: Phase Transitions and Critical Phenomena, Vol 11, Domb and Lebowitz (eds.) (1987).

5. Zahradnik, M., "Analyticity of low-temperature phase diagrams of lattice spin models." J. Stat. Phys. 47, 725-755 (1987).

6. Brydges, D. A short course on Cluster expansions. In: Osterwalder, K., Stora, B. (eds.) Critical Phenomena, Random Systems, Gauge Theories: Les Houches Session XLIII. Amsterdam: North Holland 1984.

7. $\quad$ Pirogov, S.A., Sinai, Ya. G. "Phase diagram of classical lattice systems I and II." Theor. Math. Phys. 25, 1185 and 26, 39 (1976).

8. Sinai, Ya., Theory of phase transitions: Rigorous results. New York: Pergamon Press 1982.

9. Preston, C., Random Fields. In: Lecture notes in Mathematics, Vol. 534. Berlin Heidelberg, New York: Springer 1976.

10. Lebowitz, J., Martin-Lof, A. "On the uniqueness of the equilibrium state for Ising spin systems." Commun. Math. Phys. 25, 276-282 (1972).

11. Dobrushin, R. L., Kolafa, J., Shlosman,S.B., "Phase diagram of the twodimensional Ising antiferromagnet (computer-assisted proof)." Commun. Math. Phys. 102, 89-103 (1985) 